\section{BEARING THE LOAD FOR YOUR INSTRUMENTS}

The Barden Corporation's X-life range of premium quality bearings are produced using state-of-the-art manufacturing technologies that enable a more uniform surface over the whole contact surface between the rolling elements and raceway. As a result, under identical load, there is a significant reduction in the stress conditions present on the rolling elements and raceway. This means reduced friction and lower bearing temperatures, less strain is placed on the lubricant, higher basic dynamic load ratings, and an increased basic rating life.

An important bearing feature is the use of ceramic balls rather than steel balls. Ceramic balls are harder, lighter and more wear resistant than their steel counterparts. At speeds of around $450,000 \mathrm{rpm}$, this means the ceramic balls generate less centrifugal force, which reduces wear and internal loads on the bearing. Lubricant life is also extended, since ceramic balls produce fewer wear particles than steel balls.

Barden dental bearings can be supplied with a number of different lubrication options. Bearings can be oiled, where the customer uses their own lubrication, or greased with Barden's own standard grease or with a type of grease agreed with the customer. Some dental bearings are supplied dry, when the bore is glued onto the rotor shaft. Reader response number 62

\section{FINANCIAL SOLUTIONS FOR YOUR BUSINESS}

With the government's decision to reintroduce 2012 as the start date for the introduction of automatic enrolment into employee pension schemes, many dentists may be wondering how this change in employment legislation stands to affect them financially.

Thanks to their specialised experience in offering financial advice to the dental market, Lansdell and Rose are well-placed to help dental businesses navigate this challenging area of legislation and work with them to devise a plan to manage the costs involved in joining the scheme.

Lansdell Rose provides individuallytailored guidance and recommendations based upon your unique circumstances, as well as a full complement of services to help you run a more efficient and

\section{SPECIAL CARE GUIDANCE FRAMEWORK}

The British Society for Disability and Oral Health (BSDH) guidelines on clinical holding (Unlocking barriers to care guidelines for 'clinical holding' skills for dental services for people unable to comply with routine oral health care 2009) provide a framework of practice that guides dental practitioners on the use of physical interventions for a range of dental procedures including oral, nasal and intravenous sedation, and general anaesthesia.

At the same time, Surrey PCT Special Care Dental Services have worked profitable practice, including:

- Pension planning for staff and your retirement

- Tax advice to hep you operate at maximum efficiency

- Expertise in helping you incorporate your practice

- Assistance with making strategic investments, including risk analysis and identifying the best savings schemes

- Income protection packages to help you safeguard your business and family

from critical illness, disability or death.

Why not consult Lansdell \&t Rose to find out how compulsory pension contributions stand to affect your business, as well as learn more about the available solutions for minimising the potential impact on your practice?

Reader response number 63

together with Positive Options (a national training provider) to pioneer a staff development programme on clinical holding based on the BSDH guidelines which subsequently has been delivered to dental staff within Surrey and other PCTs. At a time when value for money and patient focussed outcomes are of critical importance within the public sector, this programme provides dental staff with the necessary critical decision making skills and physical interventions for everyday oral care and treatment enabling safer practice and improved patient outcomes.

Reader response number 64

\section{ALL-IN-ONE TOOTH GUARD}

Oral-B's new all-in-one Pro-Expert toothpaste is set to bring relief to thousands of dentine hypersensitivity suffers, thanks to its inclusion of stabilised stannous fluoride. While reducing hypersensitivity is just one of the comprehensive range of oral health benefits delivered by Oral-B Pro-Expert toothpaste, for people who experience this painful condition it will probably be enough by itself.

Hypersensitivity is caused by nerves which are stimulated in the dentine layer of teeth exposed by tooth wear or periodontal disease. Stabilised stannous fluoride, researched in both laboratory and clinical studies acts by partially or completely blocking the tiny tubules of which dentine is composed.

As a multi-benefit dentifrice, the allin-one oral health advantages of OralB Pro-Expert toothpaste are not only clearly understandable for professionals. Patients who suffer from hypersensitivity will soon also appreciate its advantages in relieving this condition through advanced technological research. By improving gum health and helping to reduce the risk of erosion, Pro-Expert toothpaste will also help guard against the causes of sensitivity for future peace of mind.

Reader response number 65

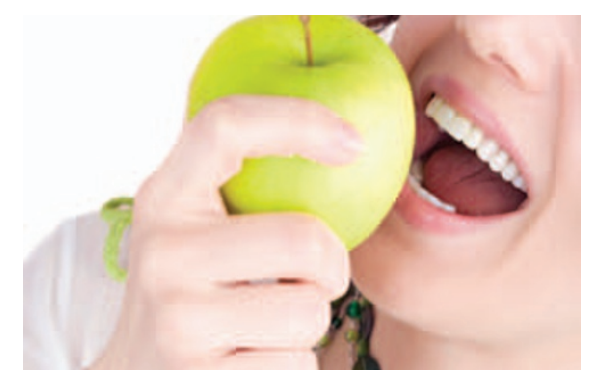

